\title{
MODELO ESTRATÉGICO DE GESTIÓN PARA INVESTIGACIÓN EN UNA INSTITUCION DE EDUCACIÓN SUPERIOR
}

\author{
JOSÉ-IRIGOYEN-BALDENEGRO \\ Instituto Tecnológico de Sonora, México \\ jirigoyen2002@yahoo.com.mx
}

\section{Resumen}

Producto de un Plan Estratégico Integrado para una Institución de Educación Superior (IES), basado en metodología de Tecnología del Desempeño Humano (HPT), se identificó problemática relativa a su gestión investigadora, contextualizada en baja productividad, poco valor agregado a la sociedad y desarticulación con las necesidades sociales; evidenciando el requerimiento de una reconfiguración estratégica y sistémica de sus procesos. En este contexto, de acuerdo a un análisis de opinión y satisfacción de investigadores institucionales y un proceso metodológico de integración, se propone un modelo estratégico de gestión con una visión social a través de tres niveles de resultados (Mega, Macro y Micro); sirviendo como marco rector de organización mediante la sinergia de estrategias, acciones y recursos vinculables a las áreas y factores críticos de éxito de la actividad investigadora institucional; garantizando su fortalecimiento y mejora continua en términos de productividad, cierre de brechas, satisfacción de usuarios y valor social.

Palabras clave: Sistemas de investigación universitarios, planeación y gestión estratégica, factores críticos de éxito, logro de visión, mejora continua.

\section{Abstract}

As a product of an Integrated Strategic Plan for Higher Education Institutions (IES), based on a Human Performance Technology (HPT) methodology, problems related to their research management were identified; problems related to low productivity, low value added to society and disentaglement from social needs show that a strategic and systemic reconfiguration of their management processes is required. In this context, according to an analysis of opinion and satisfaction of institutional researchers and to a methodological integration process, a three-level (Mega, Macro and Micro) strategic management model with a social vision is proposed; this model will serve as a framework guiding the organization through the synergy of strategies, actions and linkable resources to areas and critical success factors of institutional research activity, strengthening and ensuring continuous improvement in productivity, closing gaps, user satisfaction and social value.

Keywords: Systems of university research, planning and strategic management, critical success factors, achievement of view, continuous improvement. 


\section{INTRODUCCIÓN}

Desde sus orígenes, las IES, han tenido la misión y visión de generar conocimiento en sus diversas vertientes; de difundirlo y utilizarlo para contribuir a solucionar los retos que cotidianamente enfrentan las diversas regiones de su área de influencia e impacto; adquiriendo en este sentido, la investigación universitaria, un carácter estratégico; y por ende, manteniendo una gran responsabilidad en la búsqueda de respuestas y en la cristalización de soluciones a necesidades sociales concretas. Por lo que este contexto, a mediados de la segunda década del siglo XXI, el principal reto para las IES, consiste en proveer a la creciente sociedad demandante de las herramientas, metodologías y avances tecnológicos suficientes y pertinentes; trasladando dichos conocimientos al sector productivo para su transformación en bienes, procesos y servicios útiles; aprovechables por la sociedad, tendiente al desarrollo humano. Este contexto, de acuerdo a Ginés (2004) conduce a la definición de un nuevo modelo de universidad, caracterizado por la globalización (compitiendo en un entorno global), por la universalidad (sirviendo a todos y en todo momento), y por la necesidad de dar respuesta a las nuevas demandas de la sociedad del conocimiento.

Con base en lo anterior, a las IES, de acuerdo con Ortiz (2005) no debe bastarles con ser eficientes, sino también eficaces; ya que ambas categorías son necesarias y complementarias; por lo que hoy, más que nunca, se requiere diseñar e implementar criterios y mecanismos de calidad, gestión y organización sistémicos, para que estas instituciones respondan a las necesidades que satisfagan lo que su comunidad demanda en términos de suficiencia y pertinencia; por ello, enfatiza el autor, la implementación por parte de las IES de sistemas estratégicos y sistémicos de excelencia en materia de organización y gestión, es de suma importancia, tendiente a su consolidación, sustentabilidad y sobrevivencia. Por lo tanto, el que las IES dispongan de un plan de gestión estratégico, les permite como organización: El logro de ventajas competitivas, la racionalización en la toma de decisiones, una pertinente articulación de la misión y visión institucional, disminución de riesgos, eficiencia en el uso de los recursos, identificar los factores críticos de éxito, detectar desviaciones y operar ajustes certeros y eficaces; entre otros; consolidando estos referentes, la capacidad institucional para atender eficaz y eficientemente las señales evolutivas de su entorno, garantizado un éxito sostenido en un medio cada vez más hostil y competitivo. Circunstancia que enfatiza que el esfuerzo sistematizado de plantear y replantear estratégicamente el horizonte, deben de constituir los referentes a desarrollar y capitalizar por las universidades con visiones de excelencia.

En este contexto, se desarrolló un plan estratégico integrado para la Universidad Estatal de Sonora (UES), utilizando para tal efecto, la metodología HPT'1 (Bernárdez, 2005) que estructura tres niveles de resultados en su planeación (Mega, Macro y Micro), incluyendo cinco grandes fases tendiente a la implementación de proyectos de mejora del desempeño; comenzando con la fase de análisis del desempeño (performance), identificando las metas o resultados deseados, comparándolos con la situación actual; en este ámbito la identificación de necesidad para el nivel mega fue la siguiente: Función investigadora institucional, desarticulada con las necesidades sociales del entorno; para el nivel macro u organizacional, la necesidad identificada fue: Baja productividad investigadora institucional; $y$, por último para el nivel micro o de productos, las necesidades identificadas, fueron: carencia de un plan de organización y gestión para la actividad investigadora institucional, baja cantidad

1 HPT. Human Performance Technology. Tecnología del Desempeño Humano. (Bernárdez, 2005). 
de Cuerpos Académicos y sin consolidar e insuficiente normativa dedicada a la productividad investigadora institucional.

Esta fase permitió establecer hipótesis de solución de los problemas detectados; derivando en dos etapas principales: El análisis y agrupamiento de causas y factores y el análisis de las interrelaciones entre causas, también llamado análisis de consecuencias. Durante la presente etapa se utilizó la metodología FODA (Fortalezas, Oportunidades, Debilidades y Amenazas) identificando los referentes del ámbito interno y externo institucional para la función sustantiva de investigación. Estos resultados, impusieron la necesidad de un replanteamiento de la visión y misión institucional, en términos de innovación, impacto y pertinencia social; por lo que, bajo un nuevo enfoque, la visión quedó definida de la siguiente manera: Universidad de vanguardia, con reconocimiento internacional por su modelo de producción y socialización de conocimiento pertinente y significativo e innovador, incluyente, flexible y dinámica, con un compromiso permanente hacia la excelencia y la competitividad a través de la formación integral de profesionales en ambientes de aprendizaje centrados en la solución de problemas, comprometidos con los principios y valores de la sociedad y las necesidades del entorno.

Así mismo, un Tablero de Control Estratégico (TCE) fue implementado como herramienta metodológica de seguimiento y control a fin de permitir traducir la visión en medidas de actuación; es decir, se trasladó la visión a la acción, organizando los temas estratégicos a partir de cinco niveles (Mega, Macro, Micro, Procesos y Recursos); donde estos, integran un conjunto equilibrado de objetivos estratégicos que permitirán balancear la dirección y progreso de la institución; incluyéndose en este TCE los siguientes elementos: Los objetivos estratégicos, indicadores, metas, temporalidad, responsables y la determinación de los proyectos específicos priorizados.
En el TCE a través de su apartado de procesos, se aprobó atender prioritariamente el proyecto referido a consolidar la productividad investigadora institucional; circunstancia que da sustento al desarrollo de la tercera etapa de la Metodología HPT, referida a la selección y diseño de intervenciones; a través de la cual, se genera una propuesta de solución tecnológica, objeto del presente estudio, mediante un proyecto específico que atienda la organización y gestión de la función sustantiva de investigación institucional; procediéndose posteriormente a implementar las dos etapas siguientes, referidas a la implementación de la propuesta (intervención) y el establecimiento de un subsistema de evaluación, retroalimentación y seguimiento.

\section{Marco teórico conceptual}

A lo largo de la historia de la administración, se han desarrollado métodos e instrumentos para establecer y mejorar las normas de actuación de las instituciones e individuos que las integran; las IES no han estado ajenas a estas herramientas. En este contexto, sus perspectivas han estado vinculadas al desarrollo que ha tenido la ciencia de la administración desde sus inicios con los trabajos de los clásicos Fayol y Taylor (Fayol, 1987) hasta la tendencia más aceptada en la actualidad que es la teoría de sistemas, en la que se ve a toda institución o entidad como un sistema; donde, en función de lo estipulado por González (2005) se utiliza el enfoque sistémico en el ciclo del proceso de administración, desarrollando modelos integrales en los que se articulan los sistemas de control interno, gestión de la calidad, desarrollo administrativo y sistema de medición; acotando en este sentido González y Ayarza (1997) que las instituciones universitarias (Sistema educativo) son un sistema dentro de un sistema más complejo y dinámico (Social) caracterizadas en un proceso de intercambio permanente y constante. 
Actualmente, las formas tradicionales de planeación y gestión se han transformado radicalmente y las universidades, en términos de sus funciones sustantivas tradicionales (Docencia, investigación y extensión) tienen un gran reto por delante que sólo pueden enfrentarlo cambiando o fortaleciendo sus modelos; donde, de acuerdo a Capó (2006) el cambio de modelo o paradigma constituye un proceso de transformación en las formas de pensar y actuar que involucra a toda la comunidad universitaria en una renovación permanente de nuevos valores, estilo de actuación y de la cultura organizacional en sentido general. Por su parte, Cabeza, Cabrita \& Serey (2002) establecen que las nuevas tendencias en el financiamiento y las orientaciones de las políticas públicas han generado un cambio fundamental en la gestión y desarrollo de estas funciones sustantivas universitarias, vinculables a la mejora de su eficiencia; por lo que las IES deben adoptar innovadores sistemas de gestión, que incluyan mediciones objetivas y rigurosas de sus procesos; teniendo como consecuencia la exigencia de una actuación más activa e innovadora de las instituciones y particularmente, en las universidades públicas.

Por su parte, Royero (2003) añade que la realidad de las IES, requiere de soluciones viables a los múltiples problemas que confrontan sus procesos básicos; de los cuales, la investigación constituye un factor preponderante en la administración del sistema institucional en su papel como valor social; enfatizando que una dirección eficaz de la función de investigación debe comenzar con una programación estratégica, donde los objetivos y la estrategia sean la base para la definición de las áreas claves de gestión, con el propósito de definir los indicadores y estándares correspondientes que permitan el control de dichos procesos y la atención eficiente de los beneficiarios del sistema. En una perspectiva más específica, Puerta (2008) establece que las actividades de ciencia y tecnología tienen un enorme valor social, en la medida que son concebidas y desarrolladas desde la visión de su función multiplicadora, por lo que su gestión debe ser abordada como un asunto de interés social; observando las IES criterios de calidad y eficiencia bajo un modelo determinado de planeación estratégica para la toma de decisiones; por lo que para que dicha institución pueda considerarse como exitosa, requiere observar los principios de eficacia y eficiencia; representadas por los logros, la satisfacción del cliente (eficacia); los procesos y la organización (eficiencia).

En términos relativos a la utilización de la planeación estratégica en las instituciones, Kaufman (2005) en una visión integral, la concibe como un sistema que contiene un determinado número de procesos que hacen de ella una actividad dinámica, flexible y continua; concebida como un instrumento racionalizador en un entorno cambiante; proponiendo un esquema para su proceso dividido en cinco fases: El proceso comienza identificando las metas o resultados deseados (desempeño deseado), comparándolos con la situación actual. Las diferencias o brechas entre la situación deseada y la actual en términos de objetivos constituyen las necesidades de mejora.

Bernárdez (2005) referenciando a Roger Kaufman (2005) define que las necesidades deben comenzar a definirse desde el nivel social o Mega, partiendo de definir una misión basada en la visión ideal de la sociedad; la cual, debe ser definida en términos de indicadores medibles y alcanzables (indicadores-meta sociales); mismos que a su vez permiten definir los organizacionales (Macro) y los atributos de productos o servicios a entregar para lograrlos (Micro). La siguiente fase es la llamada análisis de causas, en la que se establecen los factores que provocan esas diferencias o brechas y su relación entre sí. La tercera fase se denomina: Selección y diseño de intervenciones, donde se consideran diversas tecnologías de intervención como medios alternativos o complementarios para resolver los problemas 
planteados. La cuarta fase es la implementación de la intervención para producir un cambio estable y sostenible en el desempeño, incluyendo el establecer un sistema para su gestión; y, la quinta y última fase consiste en el establecimiento de un subsistema de evaluación, retroalimentación y seguimiento.

\section{Justificación}

Tendiente a su pertinencia y sustentabilidad una IES requiere el logro de una visión innovadora, eficaz y socialmente responsable en términos de su función sustantiva de investigación, fundada en los principios de organización que definen a una universidad de calidad; requiriendo por lo tanto, el efectuar una reconfiguración estratégica y sistémica de sus procesos y gestión; basando su toma de decisiones en mecanismos de planeación, organización y control, eficaces, eficientes y confiables. En este contexto, la solución tecnológica propuesta proporciona un marco rector a través del cual la UES podrá normar su criterio para la toma de decisiones efectivas; operando como un modelo de organización que contribuye al fortalecimiento y mejora continua de los procesos vinculantes a la actividad investigadora institucional; garantizando la efectividad, consistencia y sinergia de las estrategias y los recursos humanos, materiales y técnicos dedicados a la investigación en términos de mejorar su productividad, calidad e impacto social.

\section{PLANTEAMIENTO DEL PROBLEMA}

Los resultados de las fases de análisis del desempeño y análisis de causas, implementadas mediante la metodología de HPT (Bernárdez, 2005) identifican necesidades (brechas) y áreas de oportunidad para la función misional de investigación institucional en función de su organización y gestión, derivando en una baja productividad investigadora, poco valor agregado a la sociedad y desarticulación con las necesidades sociales y planes sectoriales; circunstancia que la ha colocado en rezago, respecto a otras IES a nivel regional y nacional, en materia de indicadores de productividad y calidad en la generación y aplicación del conocimiento.

Lo anterior, pone en evidencia que los esfuerzos y estrategias implementadas a la fecha no han tenido la sinergia y el impacto requerido en términos de eficacia, suficiencia y pertinencia, para lograr las metas compromiso y visión institucional, en términos de su función sustantiva de investigación. En el presente contexto, se plantea la implementación de una solución tecnológica eficaz y eficiente con base en el siguiente cuestionamiento: ¿Cómo gestionar el desarrollo de las actividades de investigación en una universidad de forma integral para mejorar la generación de productos y servicios de alto impacto y valor agregado en la comunidad?

\section{OBJETIVO GENERAL}

Diseñar un modelo integral que coadyuve a desarrollar e incrementar la productividad investigadora institucional, a través de la gestión de productos y servicios de investigación, innovación y transferencia tecnológica de alto impacto y con valor agregado a la sociedad.

\section{MATERIALES Y MÉTODOS}

\section{ANÁLISIS DE OPINIÓN PARA IMPLEMENTAR LA PROPUESTA DE SOLUCIÓN TECNOLÓGICA.}

a) Población sujeto de estudio: Profesores de Tiempo Completo (PTC) investigadores, con una muestra válida de 59. b) Materiales utilizados: Cuestionario conformado por 11 ítems de cuestionamiento. c) Métodos, técnicas e instrumentos para recabar información: Métodos utilizados: De encuesta; carácter y tipo de la información: Cuantitativa y cualitativa; técnica para recabar información: Cuestionario (Abierto y cerrado). 


\section{ANÁLISIS DE SATISFACCIÓN DE LA GESTIÓN INVESTIGADORA INSTITUCIONAL}

Se implementó un cuestionario estructurado con 5 referentes de cuestionamiento a través de 4 opciones de respuesta mediante la escala de Likert ; aplicándose a 59 PTC investigadores. Se utilizó el coeficiente Alfa de Cronbach para validar la confiabilidad del instrumento aplicado mediante el uso del software SPSS versión 19.

\section{INTEGRACIÓN DEL MODELO DE SOLUCIÓN TECNOLÓGICA}

El modelo se implementó en el contexto de un orden procesal (véase Tabla 1), mediante una serie de fases programáticas con base en una adaptación de la metodología planteada por Lagarda (2010).

\section{RESULTADOS}

\section{REFERENTE DE OPINIÓN PARA IMPLEMENTAR LA PROPUESTA DE SOLUCIÓN TECNOLÓGICA}

Se establecen a continuación para efectos de dimensionamiento integral, tres resultados de referencia en relación al cuestionario aplicado; mismos que identifican los aspectos medulares tendiente a la opinión de la población y la consiguiente factibilidad de implementar la solución tecnológica propuesta (modelo); procediendo finalmente a un análisis integral.

a) En relación al grado en que la función misional de investigación institucional, está cumpliendo con su cometido e impacto social, el 100\% de los PTC investigadores, mantuvieron una percepción deficitaria en este contexto; estableciéndose su percepción por debajo del 40\%; circunstancia apreciable a través de la Figura 1.

TABLA 1

\section{FASES PARA INTEGRACIÓN DEL MODELO PROPUESTO}

\begin{tabular}{c|l|l|l} 
Fase & Denominación de la fase & \multicolumn{1}{|c|}{ Referente de actividad } & \multicolumn{1}{c}{ Métodos, técnicas e instrumentos } \\
\hline 1 & Fase de sensibilización & $\begin{array}{l}\text {-Diagnóstico del contexto nacional e internacional de investiga- } \\
\text { ción universitaria (Indicadores, modelos, logros e impactos) } \\
\text {-Diagnóstico de buenas prácticas de la IES líderes en materia de } \\
\text { investigación universitaria. } \\
\text {-Análisis de metas compromiso, estrategias e indicadores } \\
\text { institucionales en materia de investigación. } \\
\text {-Análisis de brechas, FODA, visión y misión. } \\
\text {-Reuniones informativas y mesas de trabajo }\end{array}$ & $\begin{array}{l}\text { Mécnica documental: Bases de datos físicas y } \\
\text { digitales. } \\
\text { Metodología FODA. } \\
\text { Metodología HPT (Mega planeación) } \\
\text { Sesiones informativas presenciales y virtuales. }\end{array}$ \\
\hline 2 & Fase de conceptualización & $\begin{array}{l}\text {-Diseño, evaluación y consolidación de criterios y conceptos } \\
\text { aplicables a la función sustantiva de investigación institucional. } \\
\text {-Reuniones de análisis }\end{array}$ & $\begin{array}{l}\text { Mesas de trabajo. } \\
\text { Sesiones informativas presenciales y virtuales. }\end{array}$ \\
\hline 3 & Fase del diseño del modelo & $\begin{array}{l}\text { •Estructuración del modelo por parte del consultor. } \\
\text {-Reuniones de avance informativo y ajustes. } \\
\text {-Versión final del modelo. }\end{array}$ & $\begin{array}{l}\text { Reuniones presenciales o virtuales: Informes de } \\
\text { avances, ajustes, análisis, propuestas y libera- } \\
\text { ción de apartados. }\end{array}$ \\
\hline 4 & Fase de validación del \\
modelo & -Presentacióny validación de modelo. & Documento oficial \\
\hline
\end{tabular}

Fuente: Elaboración propia con base en modelo de Lagarda (2010). 
FIGURA 1

\section{EVALUACION DE PTC INVESTIGADORES SOBRE: MISION INSTITUCIONAL Y SU GRADO DE IMPACTO SOCIAL}

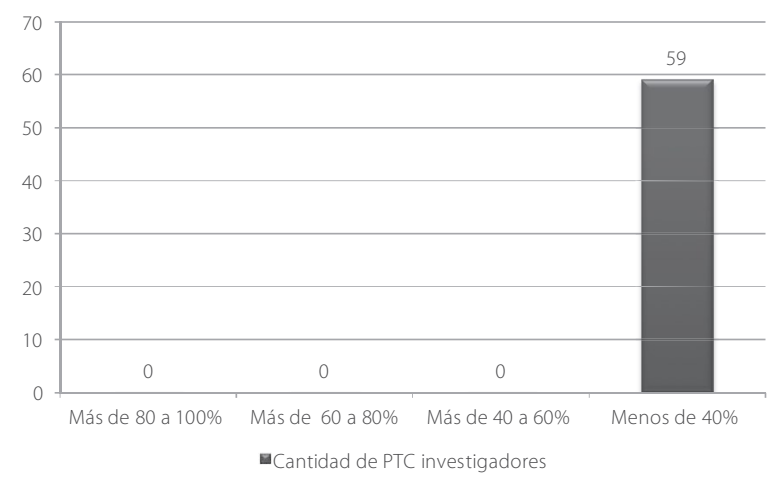

Fuente: Elaboración propia

b) En consideración al grado en que se establece como importante la implementación y uso de indicadores en la gestión y procesos de la actividad investigadora institucional, la concepción de la planeación y control como elementos nodales para lograr resultados óptimos en la gestión de investigación institucional, quedó manifiesta en función de que en el presente rubro de análisis, el 100\% de los PTC investigadores (Véase Figura 2), consideraron la implementación de indicadores de desempeño como las herramientas que fortalecen y orientan la toma de decisiones asertivas, basadas en referentes objetivos, válidos y legitimados institucionalmente; estructurados, en torno a las áreas y factores de éxito identificables, institucionalmente.

c) En el contexto de la priorización de factores correlacionados al incremento de eficiencia, eficacia e impacto de los productos y servicios de investigación, innovación y transferencia de tecnología, institucional; el 100\% de los encuestados (Obsérvese Figura 3), se inclinaron en considerar que el principal factor indexado en este contexto, es el referido al diseño e implementación de un sistema estratégico integral de planeación, organización y control de la actividad investigadora institucional.
FIGURA 2

\section{EVALUACION DE PTC INVESTIGADORES SOBRE: IMPOR- TANCIA DE INDICADORES DE GESTION Y PROCESOS EN LA ACTIVIDAD INVESTIGADORA INSTITUCIONAL}

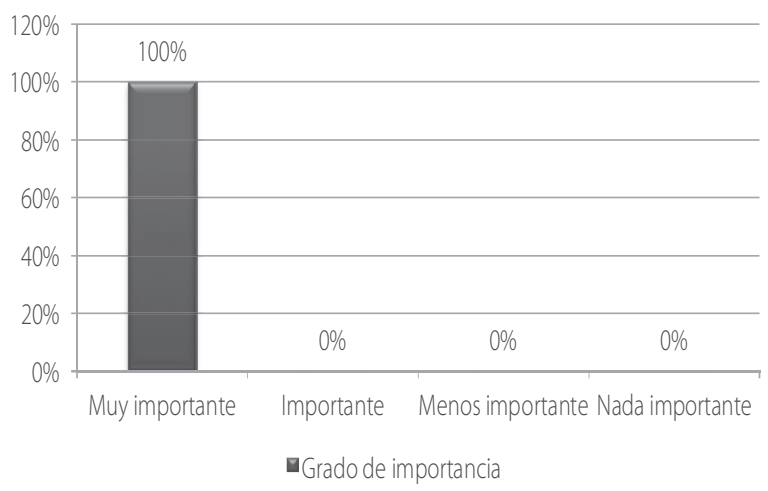

Fuente: Elaboración propia

En un análisis integrador, se evidenció que los PTC investigadores reconocen y manifiestan que la actividad investigadora institucional, se mantiene actualmente en niveles de rezago y déficit operacional; en términos de su productividad, pertinencia, consistencia e impacto social; urgiendo, la priorización de atender dicha actividad en un contexto integral, sistémico y estratégico; implementando mecanismos eficaces de planeación, dirección y control de los recursos humanos, materiales y técnicos que intervienen en esta función sustantiva.

El factor señalado como debilidad priorizada es el referido a la carencia de un sistema de planeación estratégica para el área de investigación; circunstancia que valida la implementación de un modelo de organización y gestión que atienda esta debilidad priorizada para el sistema de investigación institucional. En este contexto, en una visión ejecutiva de análisis a la información recolectada, se identifican los siguientes concluyentes:

a. Hay un dimensionamiento integral en referencia a la problemática priorizada y su eventual solución a través de una organiza- 


\section{EVALUACION DE PTC INVESTIGADORES SOBRE: FACTORES RELACIONADOS A IMPACTO Y PRODUCTIVIDAD INVESTIGADORA INSTITUCIONAL}

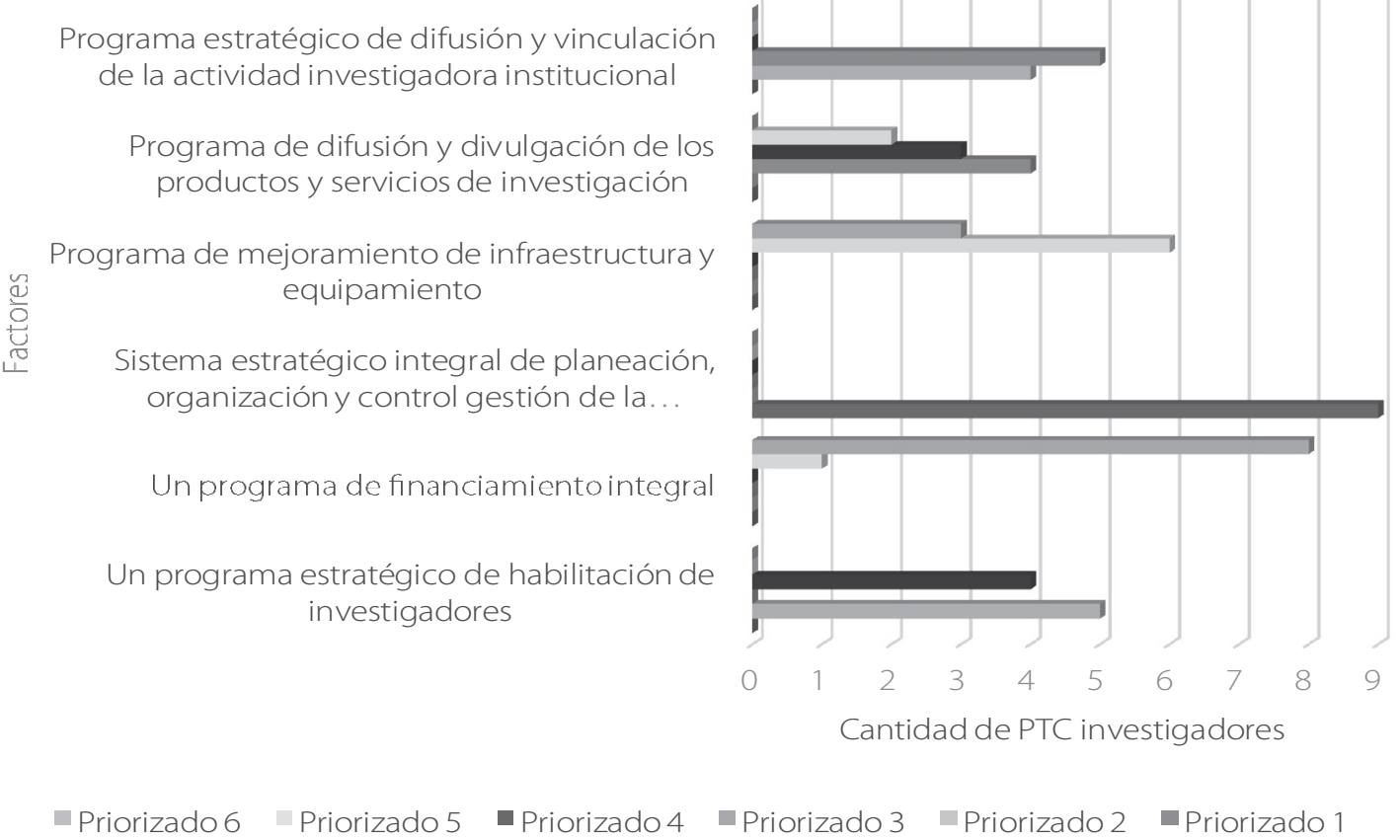

Fuente: Elaboración propia

ción sistémica y estratégica en la actividad investigadora.

b. Se presenta la condición coyuntural que permite implementar un eventual programa de organización estratégica para la función sustantiva de investigación.

c. Se identifica un cambio paradigmático de cultura institucional en la consideración de que para lograr mayores y mejores rendimientos, se debe ejercer una mejor organización estratégica y sinérgica de los recursos disponibles.

d. Se considera la implementación de indicadores de desempeño como las herramientas que fortalecen y orientan la toma de decisiones asertivas; basados en torno a las áreas y factores de éxito.

e. Se reconoce a los elementos de planeación, organización, dirección y control, como los referentes fundamentales que implementan estratégicamente las IES, que son reconocidas por la sociedad y los organismos especializados a nivel nacional e internacional.

\section{REFERENTE DE SATISFACCIÓN DE LA GESTIÓN INVESTIGADORA INSTITUCIONAL}

En este contexto, el 77.15\% de los encuestados, consideraron con deficiencia el desempeño actual de la actividad investigadora institucional, presentando grandes áreas de oportunidad, 
manifestando a su vez un $65.71 \%$ de los encuestados que perciben que la función de investigación no esta cumpliendo con su cometido e impacto social; circunstancia que a su vez atribuyen a los mecanismos y estrategias implementados actualmente por la institución, al manifestarse en total desacuerdo un $62.86 \%$ y $17.14 \%$ en desacuerdo.

Con base en lo anterior un gran porcentaje (87.15\%) de los encuestados, mencionan que las IES consideradas de excelencia mantienen como premisa el basar su toma de decisiones en un modelo oficial para la gestión de su actividad investigadora; refrendando la presente condición casi el total de los mismos, al establecer que la gestión de la actividad investigadora y su impacto mejoraría en un contexto de planeación y organización estratégicos; priorizando el 100\% de ellos, la conveniencia e importancia del uso de indicadores para efectos de la evaluación, control y mejoramiento continuo de la gestión. Recomendando a su vez una serie de factores, tendiente a fortalecer la gestión y organización de la actividad investigadora institucional; destacando: Un sistema estratégico integral; operación y gestión en un contexto de planeación y organización estratégica; normativa aplicable a investigación; implementación y desarrollo de centros de investigación. Por último, el coeficiente Alfa de Cronbach total del instrumento fue de .843; considerado aceptable de acuerdo al criterio preestablecido por Celina \& Campo (2005).

\section{Modelo de solución Tecnológica propuesto}

La propuesta de solución tecnológica toma su fundamento en la fusión de elementos integradores, relativos a los siguientes modelos: Modelo Integrado de Control de Gestión para sistemas de investigación universitarios, generado por Royero (2002); Modelo GIA (Ortiz \& Chaparro, 2006); y, Modelo para el Desarrollo de la Gestión del Conocimiento en los Centros de Investigación de las Universidades Públicas Colombianas (González, 2009). Los componentes del modelo planteado, establecen una serie de referentes de proceso y método; integrando seis fases identificables cronológica y metodológicamente, tendiente al logro de un proceso estratégico de planeación, organización y control de la función investigadora institucional.

En una dimensión general del modelo propuesto, este inicia a partir de un diagnóstico y de la planeación disponible institucional, consistentes con su misión y visión (mega plan); identificardo las áreas críticas que delimitan el éxito de la actividad investigadora, así como las variables y procesos involucrados; para posteriormente establecer una serie de indicadores referenciados por área y factores críticos de éxito; permitiendo su control, evaluación y planeación cíclica mediante el uso de instrumentos específicos de control y medición; abordando a su vez mecanismos e instrumentos para presentación de informes periódicos tendiente a la toma de decisiones. Por último, el modelo se fortalece con una fase relativa a la implantación de sistemas de control, mediante el análisis y adecuación de los siguientes elementos involucrados en este proceso: Los directivos del sistema, la adaptación de la estructura y el cambio cultural.

El modelo se constituye como un mecanismo estratégico a efecto de lograr pertinentemente el objetivo de investigación institucional; convirtiéndose en un instrumento rector a través del cual se garantice que las estrategias, acciones y recursos, sean eficaz y eficientemente dirigidos hacia los aspectos claves del éxito de la productividad investigadora, satisfacción de los usuarios y el logro de un aporte y valor social. En este contexto, se establecen a continuación los referentes que identifican la presente propuesta de solución tecnológica: 
a) Definición del modelo: Modelo Integral de Gestión y Control de la Investigación (MIGCl).

b) El modelo propuesto en el marco de la cadena de valor institucional: El modelo subyace alineado al proceso clave institucional: Desarrollar investigación, innovadora, significativa y de calidad (Véase Figura 4).
FIGURA 4

CADENA DE VALOR INSTITUCIONAL
NECESIDADES DE LOS USUARIOS

\begin{tabular}{|l|}
\hline \multicolumn{1}{|c|}{ PARTES } \\
INTERESADAS \\
VINCULADAS: \\
Estudiantes \\
Profesores \\
Funcionarios \\
Proveedores \\
Egresados \\
Padres \\
Gobierno \\
PADRES \\
AFECTADAS \\
VINCULADAS: \\
Empresarios \\
Generaciones \\
futuras
\end{tabular}

Fuente: Elaboración propia c) Componentes del modelo: El modelo MIGCI se estructura en seis fases; las cuales pueden ser apreciadas con detalle a través de la Figura 5.

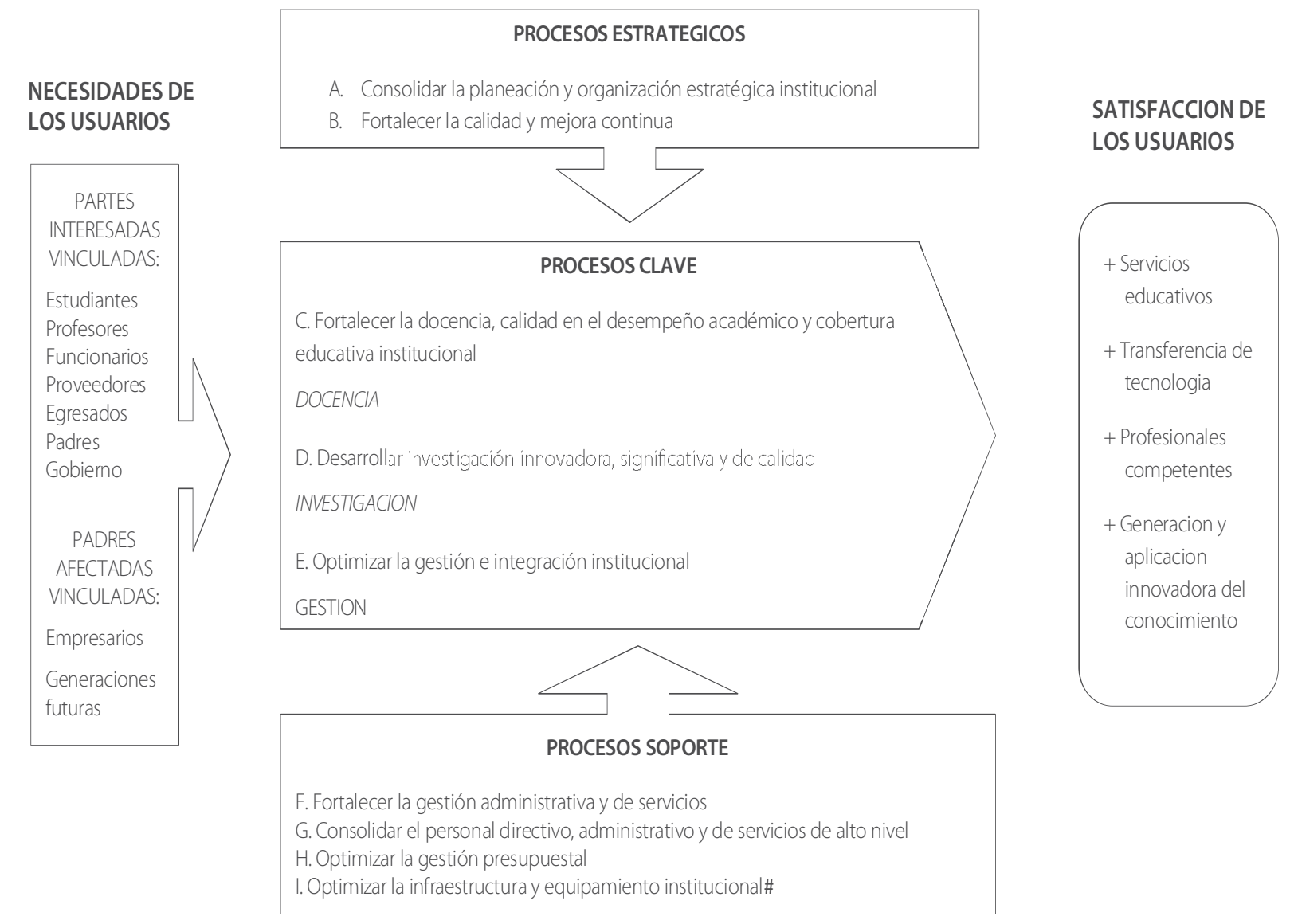




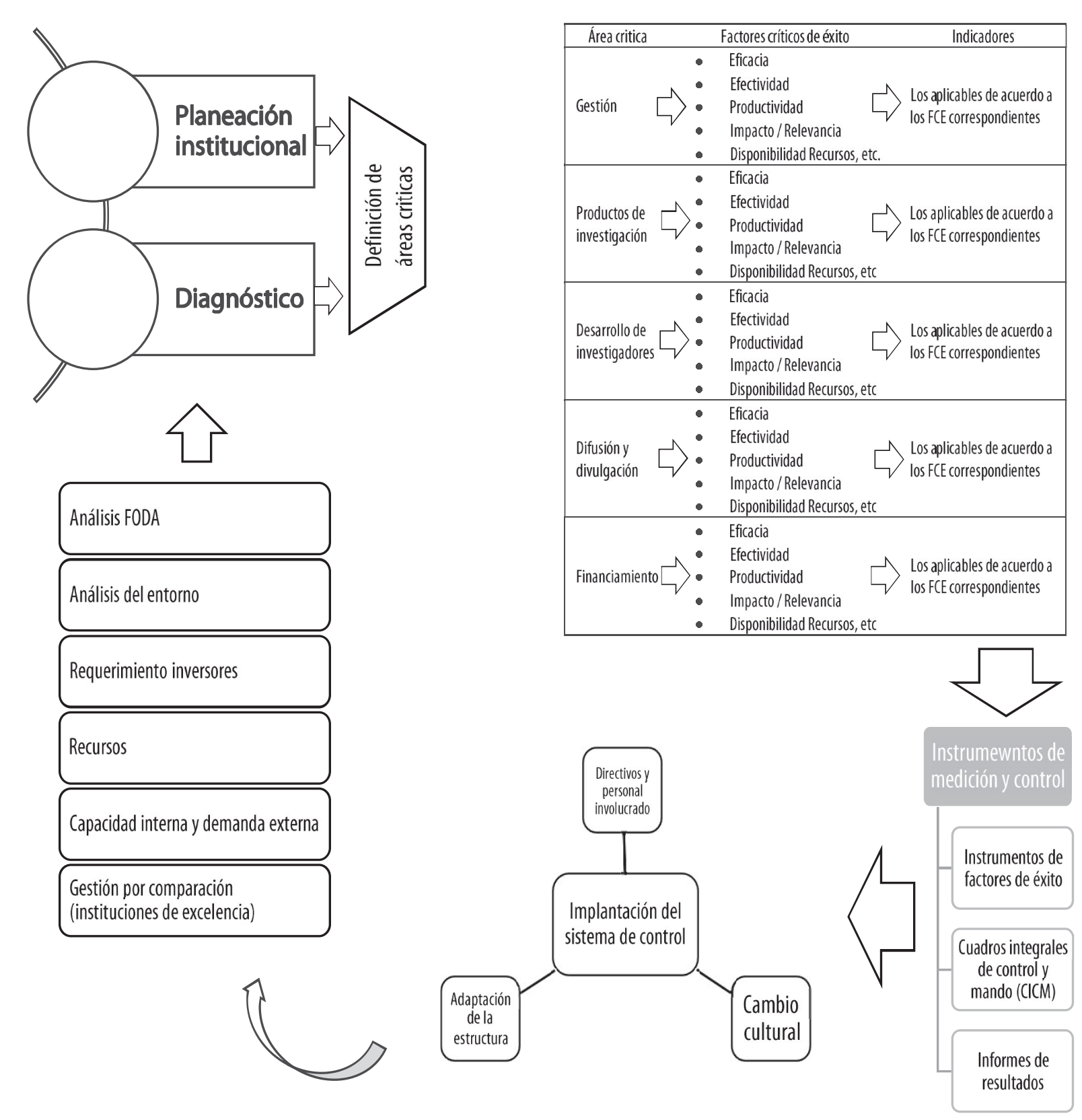

FIGURA 5

MODELO MIGCI 


\section{1) Planeación institucional pertinente y vinculante}

La planeación establecida y disponible de la institución constituye el punto de partida del modelo, tendiente a la organización estratégica institucional; en este contexto, se parte a través de un Plan de Desarrollo Institucional o de Mediano Plazo (seis años); donde este plan, define claramente los objetivos, las estrategias y las actividades del área de investigación institucional; las cuáles se mantienen debidamente alineados con los referentes de investigación, estipulados a través del Plan Nacional del Desarrollo (PND) 20132018, el Programa Sectorial de Educación (PSE) 2013-2018 y el Plan Estatal de Desarrollo (PED) 2009-2015. En este contexto, la Tabla 2, se muestra la alineación y consistencia de la planeación institucional para el área de investigación que debe regir en relación al PND 2013-2018, el PSE 2013-2018 y el PED-UES 2009-2015; a través de los ejes nacionales, objetivos estratégicos nacionales, estatales e institucionales.

La información establecida, revela la alineación y consistencia, de los objetivos y estrategias, integradas en el Plan de Desarrollo Institucional para el área de investigación; debiendo indicar una meta compromiso correspondiente al diseño e implementación de un programa de desarrollo de la investigación institucional; misma que otorgará consistencia y legitimación para la implementación del presente modelo MIGCl.

Determinada la planeación existente y su debida consistencia, se desarrolla conjuntamente la implementación de un diagnóstico institucional, basado en el modelo de elementos organizacionales de Kaufman (2005) a través de las fases de análisis del desempeño y de causas; en este contexto, los referentes de base a considerar son: El análisis FODA; un análisis del entorno institucional; requerimientos de los inversores (Sociedad, gobierno, empresarios, industriales, comunidad internacional); la capacidad interna y demanda externa institucional; y, una gestión por comparación con otras IES nacionales de excelencia. Lo anterior, permite la conjunción informativa de la planeación institucional disponible y el diagnóstico practicado a efecto de identificar un objetivo y alcance pretendido en el marco de una visión y misión institucional renovada.

\section{2) Identificación de áreas y factores críticos de éxito}

a) Identificación de áreas críticas de éxito. Constituyen el elemento clave para la ejecución del control de gestión, en los términos esperados. La identificación de las área críticas institucionales debe sustentarse a través de un mapa de procesos, elaborado específicamente para la institución, lo que permite identificar el nivel operativo del sistema a controlar a través del análisis de las actividades de transformación institucionales (insumos en productos y/o servicios institucionales); dividiéndose estas en tres elementos concretos (Figura 4): los procesos estratégicos, clave y de soporte institucionales.

En este contexto, el proceso clave institucional, denominado: Desarrollar la investigación, innovadora, significativa y de calidad, se identifica como el área crítica general, objeto de abordaje a través del presente modelo propuesto; debiendo derivar a su vez en forma específica las siguientes áreas críticas para el logro del presente proceso: Productos de investigación, gestión, desarrollo de investigadores, difusión y divulgación y financiamiento; en este sentido a su vez, la determinación de las presentes áreas, deben ser vinculantes con el objetivo estratégico institucional y los objetivos estratégicos del PED-UES 2009-2015, PSE 2013-2018 y el PND 2013-2018 (Tabla 2).

b) Identificación de los factores críticos de éxito (FCE). Identificadas las áreas críticas, se procede a determinar lo que se pretende controlar en 
dichas áreas; enfocándose el control de la gestión en los procesos críticos institucionales para el área de investigación; es decir, en los aspectos vitales que garantizan el éxito de su visión (Eficacia, eficiencia, efectividad, cobertura, pertinencia, impacto, calidad, etc.); los FCE identificables, generan implicaciones en la institución, precisando la mejora de los sistemas actualmente establecidos en la misma o bien, sugiriendo la creación de sistemas, mecanismos o elementos totalmente nuevos. En este contexto, ya validados los FCE, el paso siguiente, es el de la incorporación de indicadores clave y la asignación de responsables a través de la estructura establecida o en su caso propuesta, tendiente a verificar su realización y grado de cumplimiento.

\section{3) Diseño del sistema de indicadores}

Determinadas las áreas críticas y FCE, se asigna para cada uno de ellos, indicadores de medición en concordancia con las metas fijadas; en este sentido, los indicadores deben cumplir con las características de ser medibles, relevantes y

\section{TABLA 2}

\section{ALINEACION Y CONSISTENCIA DE OBJETIVOS Y ESTRATEGIAS DE INVESTIGACION INSTITUCIOANL PARA LA IUS}

\begin{tabular}{|c|c|c|c|}
\hline $\begin{array}{c}\text { Eje, objetivos, estrategias } \\
\text { y plan de acción }\end{array}$ & $\begin{array}{c}\text { SEP. } \\
\text { Objetivo estratégico: }\end{array}$ & $\begin{array}{l}2015 \\
\text { Objetivos estratégicos: }\end{array}$ & Objetivos estratégicos: \\
\hline $\begin{array}{l}\text { Eje 3: Educación con calidad. Plan de } \\
\text { acción 3.2: Articular la educación, la } \\
\text { ciencia y el desarrollo tecnológico } \\
\text { para lograr una sociedad más justa } \\
\text { y próspera; manteniendo como } \\
\text { premisa el que la nación dirija } \\
\text { sus esfuerzos para transitar hacia } \\
\text { una Sociedad del Conocimiento; } \\
\text { fortaleciendo en este sentido, la } \\
\text { articulación entre niveles educativos } \\
\text { yel quehacer científico, el desarrollo } \\
\text { tecnológico y el sector productivo; y, } \\
\text { teniendo como objetivo el desarrollo } \\
\text { científico, tecnológico y la innova- } \\
\text { ción, como pilares para el progreso } \\
\text { económico y social sostenible. }\end{array}$ & $\begin{array}{l}\text { Objetivo 6: Impulsar la edu- } \\
\text { cación científica y tecnológica } \\
\text { como elemento indispensable } \\
\text { para la transformación de } \\
\text { México en una sociedad del } \\
\text { conocimiento. }\end{array}$ & $\begin{array}{l}\text { 3.1.8. Convertir al Estado de Sonora } \\
\text { en un polo de investigación y desa- } \\
\text { rrollo tecnológico. } \\
\text { 3.1.9. Impulsar los intercambios y es- } \\
\text { tancias internacionales asegurando } \\
\text { un crecimiento y fortalecimiento en } \\
\text { el desarrollo integral del estudiante, } \\
\text { investigador y docente. } \\
\text { 3.1.11. Coadyuvar en los esfuerzos de } \\
\text { los institutos de educación superior } \\
\text { y el sector productivo con el fin de } \\
\text { desarrollar tecnología para generar } \\
\text { valor agregado a las materias primas } \\
\text { que se producen en el estado de } \\
\text { Sonora. }\end{array}$ & $\begin{array}{l}\text { Desarrollo de la investigación: Impulsar la } \\
\text { planeación y organización de la investi- } \\
\text { gación institucional, definiendo criterios } \\
\text { y estableciendo lineamientos para su } \\
\text { óptimo desempeño y efectividad. } \\
\text { Implementar mecanismos de fomento a } \\
\text { la productividad de investigación insti- } \\
\text { tucional. } \\
\text { Impulsar la vinculación de la investigación } \\
\text { con los diversos sectores e instituciones } \\
\text { internacionales, tendiente al desarrollo de } \\
\text { proyectos conjuntos. } \\
\text { Implementar sistemas de información y } \\
\text { servicios de apoyo para el desarrollo de la } \\
\text { investigación. } \\
\text { Propiciar condiciones de infraestructura } \\
\text { idóneas para el desarrollo de la investiga- } \\
\text { ción, que permitan mejorar los niveles de } \\
\text { calidad e innovación del trabajo cientííco } \\
\text { institucional }\end{array}$ \\
\hline
\end{tabular}

Fuente: Elaboración propia 
vinculantes; siendo parte central del proceso de evaluación y seguimiento institucional.

Con base en lo anterior, la identificación, diseño, procesamiento, monitoreo y análisis de indicadores constituyen actividades esenciales en el proceso de evaluación del modelo; consistiendo su papel en medir los resultados de la gestión institucional; en este sentido, estos indicadores deben partir de los objetivos estratégicos (áreas críticas) y de los FCE.

Mediante estos indicadores se pretende que en lo general: Sirvan de instrumentos para evaluar hasta que punto se están logrando los objetivos estratégicos institucionales; representen una unidad de medida para evaluar el desempeño de la institución frente a sus metas compromiso; produzcan información para analizar el desempeño y cumplimiento de objetivos en términos de resultados; detecten y prevean desviaciones en el logro de los objetivos; y, generen alertas sobre la acción para mantener la dirección alineada con respecto al plan estratégico establecido; impulsen los esfuerzos para medir los resultados en el abatimiento de los principales rezagos institucionales y el impacto social de las acciones realizadas en el ámbito de la investigación; y, disponer de información válida para apoyar la toma de decisiones con un sentido estratégico.

\section{4) Diseño de los instrumentos de control}

Estructurados los indicadores se procede a la asignación, para cada área crítica, de un cuadro de mando que permitirá monitorear los avances del comportamiento de los resultados de gestión; es decir, con un indicador que integre información relativa con su condición actual (condición histórica) y su meta. Los indicadores serán determinados de acuerdo a sus características históricas, reales y potenciales de cada área o factor crítico; bajo una visión integrada con los objetivos, metas y estrategias institucionales. La Figura 6, muestra el esquema correspondiente al Cuadro de Control de Mando (CCM) a utilizar como instrumento de control en el presente modelo; mismo que identifica contextualmente la relación de indicadores establecidos por cada una de las áreas críticas identificadas para la institución; estableciendo el objetivo, estrategia a que corresponde y el responsable de su monitoreo y resultados; mostrando a su vez, la relación de indicadores que se vinculan con un área crítica en particular; revelando su situación actual y su perspectiva de logro (meta).

Integrados los CCM, según correspondan a las áreas críticas, se procede a diseñar el instrumento donde se visualizará el avance real de los resultados, luego de la frecuencia definida;

FIGURA 6

CUADRO DE CONTROL DE MANDO (CCM)

\begin{tabular}{|c|c|c|c|}
\hline & \multicolumn{2}{|c|}{ Cuadro de Control de Mando } \\
\hline Área crítica: & & \\
\hline Objetivo: & & \\
\hline Estrategia: & & & \\
\hline Responsable: & & & \\
\hline Factor de éxito & Indicador & Situación actual & Meta o umbral \\
\hline & & & \\
\hline
\end{tabular}

Fuente: Elaboración propia. 
denominado: Cuadro de Mando Integral (CMI), mismo que se muestra en la Figura 7. Este instrumento permite identificar la relación de FCE establecidos por cada una de las áreas críticas identificadas para la institución; revelando la situación actual de los mismos, su perspectiva de logro (meta); así como el grado de cumplimiento que se obtuvo para un período específico.

\section{5) Presentación de informes de resultados}

Definido el CMI, se procede a presentar los resultados de manera gráfica de acuerdo a los requerimientos institucionales a fin de visualizar integralmente el comportamiento de las áreas críticas de la función sustantiva de investigación.

\section{6) Implantación del sistema de control}

Se determina los términos en que el modelo será implantado en la institución; es decir, qué recursos se necesitarán y qué cambios culturales condicionarán su aplicación. El modelo no debe de constituirse como un proceso técnico aislado a desarrollar; sino que requiere del apoyo directivo, financiero y estructural de la institución para que sea implantado con éxito en el sistema integral; en este contexto, se deberán considerar tres aspectos fundamentales.

a) Los directivos del sistema. Juegan un papel preponderante en la conducción de los procesos de cambio, ya que tienen el poder y facultad de tomar decisiones; en este sentido, deben asumir una posición participativa, continua y responsable dentro del diseño e implementación del modelo; ya que en lo general, la comunidad universitaria presenta resistencia al cambio y eventual pesimismo en función de nuevas iniciativas que se propongan por parte de los directivos; con base en lo anterior, se establece conveniente el que generen un clima de apertura institucional, asuman una postura decidida y firme en torno al modelo a seguir, incorporen a toda la

FIGURA 7

\section{CUADRO DE MANDO INTEGRAL (CMI)}

\begin{tabular}{|c|c|c|c|c|}
\hline \multicolumn{4}{|c|}{ Área Crítica: } & \\
\hline \multicolumn{4}{|c|}{ Objetivo: } & \\
\hline \multicolumn{4}{|c|}{ Estrategia: } & \\
\hline \multicolumn{4}{|c|}{ Responsable: } & \\
\hline \multicolumn{4}{|c|}{ Frecuencia de medición: } & \\
\hline $\begin{array}{l}\text { Resultados } \\
\text { Factores }\end{array}$ & $\begin{array}{c}\text { Situación } \\
\text { actual }\end{array}$ & $\begin{array}{l}\text { Meta } 0 \\
\text { umbral }\end{array}$ & $\begin{array}{c}\text { Valor } \\
\text { obtenido }\end{array}$ & Grado de Cumplimiento ** \\
\hline \multicolumn{5}{|l|}{ Eficacia } \\
\hline \multicolumn{5}{|l|}{ Efectividad } \\
\hline \multicolumn{5}{|l|}{ Cobertura } \\
\hline \multicolumn{5}{|l|}{ Pertinencia } \\
\hline Otros & & & & \\
\hline
\end{tabular}

* Estos indican los elementos involucrados en la composición específica de cada indicador para cada área crítica.

** Se calcula dividiendo el valor obtenido entre el valor meta (umbral).

Fuente: Elaboracion propia con base en modelo de Royero (2002). 
población en la mejora continua del modelo y asuman la planeación estratégica como un valor y responsabilidad.

b) La adaptación de la estructura. La institución debe contar con una estructura que facilite la implementación del modelo; determinando la jerarquización, responsabilidades, autoridad y el tipo de estructura.

c) El cambio cultural. El modelo, requiere de un mecanismo contextual que facilite su implementación. Dicho mecanismo atenderá a la cultura general y de planeación en la institución; así como la resistencia al cambio. El presente mecanismo, implica un esfuerzo de atención conjunta institucional, instrumentando diversos mecanismos informativos y de sensibilización, exponiendo los beneficios de la implantación del modelo propuesto; vinculables a la solución de problemática detectada, aprovechamiento de oportunidades, incremento de indicadores y cumplimiento de metas compromiso. Un elemento importante es la creación y mantenimiento de un clima favorable institucional; vinculando el modelo a todas las funciones sustantivas institucionales, enmarcándose como un proyecto institucional de tal manera que toda la comunidad lo asuma en términos de su quehacer y compromiso.

\section{CONCLUSIONES}

El modelo atiende al cierre de brechas identificables para la actividad investigadora institucional. El objetivo, estructura y procesos del modelo, mantienen consistencia y pertinencia en función de la contribución al logro de los objetivos estratégicos y metas establecidas para el sistema de investigación institucional, vinculables a planes sectoriales; y por ende, en el alcance de la misión y visión institucional. El modelo se estructura con la finalidad de servir como marco rector de la toma de decisiones institucionales en materia de investigación; coadyuvando al fortalecimiento de la gestión, incrementando la productividad investigadora y satisfaciendo la comunidad de impacto. El modelo propuesto involucra una visión social, a través de un valor añadido a la sociedad; contribuyendo al bienestar y mejoramiento de la calidad de vida; alineando los tres niveles de resultados de su planeación (Mega, Macro y Micro) en función de una visión ideal de la sociedad. El modelo incorpora a la planeación y gestión estratégica, como elementos nodales en el fortalecimiento y mejora continua de la función sustantiva de investigación institucional.

\section{REFERENCIAS}

Bernárdez, M. (2005). Tecnología del Desempeño Humano. Recuperado de: http://www.expert2business.com/itson/TDHbernardez.pdf.

Cabeza, Cabrita \& Serey. (2002). Algunas consideraciones sobre los indicadores de gestión en la educación superior venezolana. Revista Economía No. 17-18, 2001- 2002. (EneroDiciembre) 33-48.

Capó, J. (2006). Dirección de los procesos universitarios. Modulo II. Diplomado en

Dirección Universitaria. Universidad Agraria de la Habana. Cuba.

Fayol, H. (1987). Administración Industrial y General. El Ateneo Editorial. Ed. 14.

Ginés, M. (2004). El cambio educativo en la sociedad del conocimiento. Revista Iberoamericana de educación. No. 35 mayo-agosto.

González, J. (2009). Modelo para el Desarrollo de la Gestión del Conocimiento en los Centros de Investigación de las Universidades Públicas Colombianas. Caso Aplicativo Universidad Pedagógica y Tecnológica de Colombia. Revista Gestión y Estrategia No. 35. Enero/Junio 2009.

González. L. \& Ayarza, H. (1997). Calidad, evaluación institucional y acreditación en la educación superior en la región Latinoamericana y del Caribe. Caracas CRESALC/UNESCO. 
González, O. (2005). Los sistemas de control de gestión estratégica de las organizaciones Trabajo 71. Disponible en: www.momografías.com.

Kaufman, R. (2005). Mega Planning: Practical tools for organizational" sucess. Sage publications, Inc. Thousand oaks. London, New Dheli. Publicatiosn de la Universitat Jaume.

Lagarda, E. (2010). Strategic Planning from Casual Models of the Centers of Innovation and Development as the basis for building scenarios based on ITSON's Innovation Ecosystem. Social and Organizational Performance ReviewConcepts and Research.

López, M. Mejía, J. \& Schmal, R. (2006). Un Acercamiento al Concepto de la Transferencia de Tecnología en las Universidades y sus Diferentes Manifestaciones. Disponible en: http://www. panorama.utalca.cl/dentro/2006 jul/articulo7.pdf.

Ortiz, A. (2005). Alta gerencia educativa: ¿Cómo dirigir y administrar con eficiencia? Centro de Estudios Pedagógicos y Didácticos. CEPEDID. Colombia.

Ortiz, L. \& Chaparro, J. (2006). Modelo de Gestión de Investigación Académica (GIA); http://www. adingor.es/Documentacion/CIO/cio2005/ items/ponencias/100.pdf.http://io.us.es/ cio2006/docs/000023_final.pdf.
Puerta, M. (2008). La Gerencia de Instituciones de Investigación Científico-Tecnológicas. Revista faces volumen XIX № 2.

Royero, J. (2002). Modelo de control de gestión para sistemas de investigación universitarios. Revista Iberoamericana de Educación. España. Sección Lectores.

Royero, J. (2003). Gestión de sistemas de investigación en América Latina. Revista Iberoamericana de Educación. Edición Digital, España. Sección Lectores.

UNESCO. (2005). Hacia las sociedades del conocimiento. Organización de las Naciones Unidas para la Educación, la Ciencia y la Cultura. Ed. UNESCO. Disponible en: http//unesco.org./ publications.
Recibido: 3 de julio de 2015

Aceptado: 10 de diciembre de 2015 
This document was created with Win2PDF available at http://www.win2pdf.com. The unregistered version of Win2PDF is for evaluation or non-commercial use only. This page will not be added after purchasing Win2PDF. 